\title{
Dynamique sub-picoseconde de l'interaction laser de puissance - agrégats de gaz rare : emission intense de rayons $X$ et production d'ions multichargés
}

\author{
C. Prigent, L. Adoui ${ }^{1}$, S. Dreuil, J.C. Gauthier ${ }^{2}$, O. Gobert ${ }^{3}$, E. Lamour, \\ P. Meynadier ${ }^{3}$, D. Normand ${ }^{3}$, M. Perdrix ${ }^{3}$, J.-P. Rozet et D. Vernhet
}

\author{
GPS-PIIM, Universités Paris VI et Paris VII, 2 place Jussieu, 75251 Paris cedex 05, France \\ ${ }^{1}$ CIRIL, rue Claude Bloch, BP. 5133, 14070 Caen cedex 5, France \\ ${ }^{2}$ LULI, Ecole Polytechnique, 91128 Palaiseau, France \\ ${ }^{3}$ CEA-Saclay, DSM/DRECAM/SPAM, 91191 Gif-sur-Yvette cedex, France
}

\begin{abstract}
Résumé: Lors de campagnes d'expériences réalisées sur le Laser Ultra Court Accordable du CEA/Saclay, nous avons étudié le rayonnement $\mathrm{X}$, tant qualitativement (spectroscopie et énergie moyenne des photons) que quantitativement (taux absolus et lois d'évolution), émis lors de l'interaction d'un jet effusif d'agrégats de gaz rare (Ar, $\mathrm{Kr}, \mathrm{Xe}$ comprenant entre $10^{4}$ et $10^{6}$ atomes/agrégat) avec un laser femtoseconde de puissance (éclairement jusqu'à quelques $10^{17} \mathrm{~W} / \mathrm{cm}^{2}$ ). Les résultats présentés dans ce manuscrit sont uniquement dédiés aux agrégats d'Ar pour lesquels nous avons observé un rayonnement $\mathrm{X}$ issu d'ions fortement multichargés (jusqu'à $\mathrm{l}^{\prime} \mathrm{Ar}{ }^{16+}$ ) présentant des lacunes en couches $K$. La technique de spectroscopie $X$ utilisée a permis de déterminer pour la première fois des taux absolus ainsi que les lois d'évolution de l'émission $X$ en fonction de l'ensemble des paramètres gouvernant l'interaction (intensité, polarisation, longueur d'onde et durée du pulse laser aussi bien que taille, densité et numéro atomique des agrégats).
\end{abstract}

\section{INTRODUCTION}

La possibilité de créer de nouvelles sources de rayonnement X - UV intense à l'aide d'un laser de puissance fait l'objet actuellement de nombreux travaux tant expérimentaux que théoriques : génération d'harmoniques sur des cibles gazeuses, focalisation d'un laser intense sur des cibles solides, interaction d'un laser de puissance avec un jet effusif d'agrégats... De nombreuses études expérimentales ont montré que l'interaction d'un laser intense pulsé avec des agrégats de gaz rare se manifeste par une excitation électronique intense de la matière: éjection d'électrons «chauds» [1], production d'ions très multichargés rapides [2] et présentant des lacunes en couche interne [3], émission intense (jusqu'à $10^{12}$ photons dans $4 \pi \mathrm{str}$ / impulsion laser) de rayons $\mathrm{X}$ durs (de l'ordre du keV) [4-5].

L'avantage des agrégats, qui possèdent une densité locale d'atomes proche du solide, réside dans le fait que le champ électrique peut, en première approximation, être considéré comme étant le même sur chaque atome cible (épaisseur de peau $\sim$ taille de l'agrégat) contrairement à une cible solide où des effets d'écrantage du champ laser peuvent apparaître en profondeur. Dans le cadre de nos études, nous utilisons le rayonnement $\mathrm{X}$ émis lors de cette interaction comme une sonde temporelle de l'évolution du nanoplasma créé. La spectroscopie $X$ constitue en effet une bonne observable de la dynamique de l'interaction car l'absorption des $\mathrm{X}$ est négligeable à travers un agrégat. Par ailleurs, il est observé, dans le cas des agrégats d'Ar, des espèces beaucoup plus multichargées à l'aide de la spectroscopie X (allant jusqu'à $\mathrm{l}^{\prime} \mathrm{Ar}^{16+}$ ) que lors des études en spectroscopie d'ions (au plus $\mathrm{Ar}^{10+}$ ). Cette différence est une manifestation du fait que l'émission $\mathrm{X}$ se produit sur des échelles de temps très courtes (quelques dizaines de femtoseconde) et permet donc de sonder les phénomènes d'interaction bien avant d'éventuelles recombinaisons, c'est-à-dire au plus près de l'instant initial de l'interaction.

En dehors des applications potentielles (i.e. optimisation d'une nouvelle source X ultra courte), l'intérêt de ses études est de comprendre la dynamique des processus mis en jeu lors de l'interaction. Dans ce but, il est, en particulier, nécessaire de savoir comment des électrons de valence libérés initialement par ionisation multiphotonique atteignent une énergie suffisamment élevée pour conduire, via des collisions èions, à la création d'ions multichargés possédant des lacunes en couche interne et qui se désexcitent en émettant un important rayonnement $\mathrm{X}$. 


\section{DISPOSITIF EXPERIMENTAL}

Les différentes campagnes d'expériences que nous avons réalisées ont été effectuées auprès du serveur LUCA du SPAM/DRECAM au CEA/Saclay. Cette source femtoseconde est constituée d'une chaîne laser à Saphir dopé au Titane délivrant, à un taux de répétition de $20 \mathrm{~Hz}$, des impulsions $\mathrm{IR}(\lambda=800 \mathrm{~nm})$, d'énergie modulable entre 0 et $80 \mathrm{~mJ}$ (correspondant à un éclairement maximum de $6.10^{17} \mathrm{~W} / \mathrm{cm}^{2}$ obtenu avec une parabole et permettant de descendre à $10^{15} \mathrm{~W} / \mathrm{cm}^{2}$ à l'aide d'une lentille) et d'une durée d'impulsion comprise entre 50 fs et quelques ps. Le jet d'agrégats est produit par expansion adiabatique à travers une buse conique d'un gaz rare sous pression. Cette pression amont $\mathrm{P}_{0}$ contrôle la taille de nos agrégats suivant des lois d'échelles (taille $\propto \mathrm{P}_{0}{ }^{2}$ ) établies par Hagena [6]. En ce qui concerne le rayonnement $\mathrm{X}$, le diagnostic s'effectue à l'aide $\mathrm{d}^{0}$ un système de détection calibré en absolu et composé de 2 détecteurs $\mathrm{Si}(\mathrm{Li})$ (spectroscopie dite de «basse résolution »: de l'ordre de $150 \mathrm{eV}$ à $3 \mathrm{keV}$ ) et d'un spectromètre à cristal de Bragg de haute résolution (de l'ordre de $1,5 \mathrm{eV}$ à $3 \mathrm{keV}$ ) et de grande transmission. Nous réalisons ainsi une spectroscopie fine du rayonnement $\mathrm{X}$ et nous observons notamment les transitions de type $1 s 2 p n l$ vers $1 s^{2} n l$ issues d'états de charge allant de l'Ar ${ }^{1+}$ jusqu'à

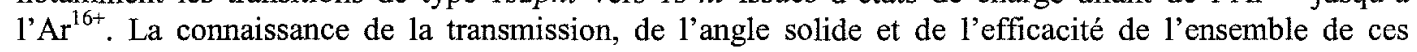
3 détecteurs permet donc de remonter à des mesures absolues de taux d'X.

\section{INFLUENCE DE L'ECLAIREMENT ET DE LA PRESSION SUR L'EMISSION X}

La technique de spectroscopie $\mathrm{X}$ utilisée permet non seulement de mesurer des taux absolus de photons $\mathrm{X}$ émis dans $4 \pi$ mais également d'étudier l'évolution de l'émission $X$ en fonction d'un, et d'un seul, paramètre; c'est à dire en contrôlant au mieux l'ensemble de tous les autres paramètres susceptibles d'influer sur l'intensité de la source $X$. Nous avons ainsi mesuré l'évolution du taux d'X en fonction de 2 paramètres importants lors de $1^{\prime}$ interaction que sont : $1^{\circ}$ ) l'intensité du champ électrique excitateur (via l'éclairement laser) et $2^{\circ}$ ) la taille des agrégats (via la pression amont). Les résultats, limités dans cet article au cas des agrégats d'Ar et obtenus à partir de l'analyse des spectres haute et basse résolution, sont les suivants :

- $\mathrm{N}_{\text {photons } \mathrm{X} / \text { pulse }} \propto \mathrm{I}_{\text {pic }}{ }^{3 / 2}$ et l'état de charge moyen $<\mathrm{Q}>$ dont est issu le rayonnement $\mathrm{X}$ est constant; ce qui est la signature d'un processus de saturation à effet de seuil. En effet, le nombre de photons X par impulsion augmente comme le nombre de partenaires émetteurs c'est à dire comme le volume focal à l'intérieur duquel une certaine intensité seuil est dépassée ;

- $\mathrm{N}_{\text {photons } \mathrm{X} / \text { pulse }} \propto \mathrm{P}_{0}{ }^{5 / 3}$ et $\langle\mathrm{Q}>$ augmente puis devient constant; ce qui est relié à une augmentation de la probabilité d'ionisation en couche interne quand la taille moyenne des agrégats augmente.

A ce stade, ces résultats obtenus à forts éclairements $\left(\right.$ de $5.10^{16} \mathrm{~W} / \mathrm{cm}^{2}$ jusqu'à $\left.6.10^{17} \mathrm{~W} / \mathrm{cm}^{2}\right)$ et pour des grandes pressions $\left(\mathrm{P}_{0}>15\right.$ bar) sont interprétés dans le cadre d'un modèle dynamique développé par l'équipe [4]. Ce modèle collisionnel repose sur un scénario s'effectuant en plusieurs étapes. Les électrons sont initialement produits, au tout début du pulse laser, par ionisation par effet de champ puis sont ensuite accélérés par ce même champ laser afin d'acquérir suffisamment d'énergie pour pouvoir enfin ioniser en couche $\mathrm{K}$ de l'Ar par des collisions de type e/ions. Les collisions inélastiques entre ces électrons et les ions, à l'intérieur même de l'agrégat et pendant son expansion hydrodynamique, correspondent au processus dominant responsable de la production de lacunes en couche interne des ions multichargés observés. Dans le cadre de ce modèle, un seuil de production $\mathrm{X}$, correspondant en fait à l'éclairement minimum pour accélérer suffisamment les électrons, est donc attendu. $\mathrm{Si}$ on considère des électrons d'énergie supérieure à $4 \mathrm{keV}$ (seuil $\mathrm{K}$ de l'Ar vers $4 \mathrm{keV}$ ), l'éclairement minimum de production d'X doit se situer vers $3.10^{16} \mathrm{~W} / \mathrm{cm}^{2}$. De nouvelles études ont donc été effectuées, en fonction de l'éclairement, afin de rechercher ce seuil de production des $\mathrm{X}$ dans le cas, par exemple, des agrégats d'argon (cf. figure 1).

Le seuil observé de production de rayons $X$ est très bas (vers $1,25.10^{15} \mathrm{~W} / \mathrm{cm}^{2}$ ) ce qui correspond, dans le cadre de notre modèle collisionel, à des énergies cinétiques d'oscillation des électrons de l'ordre de $150 \mathrm{eV}$ (!). Il semble donc que l'image d'un chauffage des électrons uniquement dans le champ laser par la force pondéromotrice de l'onde électromagnétique soit exclue. 


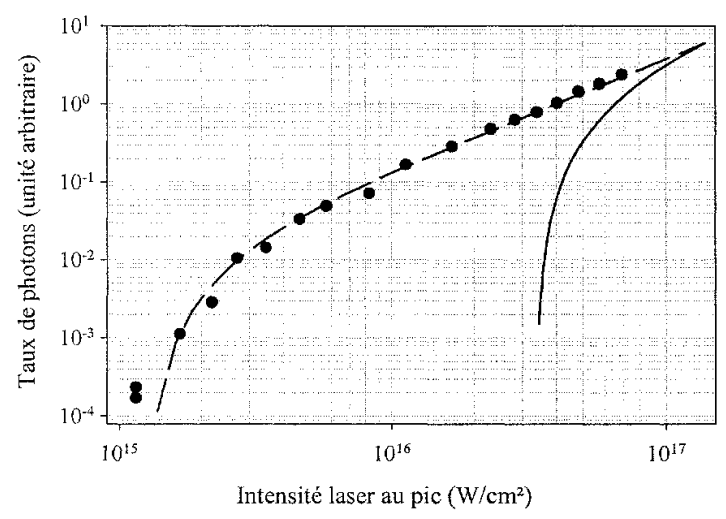

Figure 1. Evolution du taux de photons $X$ en fonction de l'éclairement laser (en IR) pour une pression de 40 bar d'agrégats d'Ar. La courbe en trait plein correspond à l'évolution attendue dans le cadre de notre modèle dynamique avec un seuil à $3,5.10^{16} \mathrm{~W} / \mathrm{cm}^{2}$. La courbe en trait pointillé correspond au meilleure fit des points expérimentaux que l'on puisse obtenir et à un seuil de $1,25.10^{15} \mathrm{~W} / \mathrm{cm}^{2}$.

Ditmire et. al. [7] évoque, dans son modèle d'expansion hydrodynamique du nanoplasma, le chauffage des électrons du plasma par une résonance qualifiée de "géante » du champ électrique à l'intérieur de l'agrégat lorsque la fréquence plasma du milieu est égal à la fréquence de l'onde incidente ; c'est-à-dire quand la densité électronique atteint 3 fois la densité critique donnée par :

$$
\mathrm{n}_{\mathrm{c}}\left(\mathrm{cm}^{-3}\right) \approx 1,11.10^{21} \lambda(\mu \mathrm{m})^{-2}
$$

Ce mécanisme d'absorption par bremstralhung inverse de l'énergie laser par les électrons, même s'il ne se produit que sur une durée très brève (de l'ordre d'un cycle optique), pourrait être responsable de la production d'électrons énergétiques pouvant aussi ioniser en couche $\mathrm{K}$ des agrégats d'Ar lors de collisions $\mathrm{e}^{-} /$ions. Le comportement du taux d'émission $\mathrm{X}$ issu de ce modèle, en fonction de l'éclairement en particulier, est actuellement à l'étude. Une compétition forte entre différents mécanismes de chauffage des électrons est tout à fait envisageable. De nouveaux résultats, encore à analyser, viennent tout juste d'être obtenu en avril 2002 concernant notamment une étude précise en spectroscopie haute résolution du seuil de production des $X$ et qui devraient nous apporter de nouvelles informations importantes concernant la formation des différentes espèces ioniques pour de faibles éclairements.

\section{EFFET DE LA LONGUEUR D'ONDE ET DE LA POLARISATION SUR LE RAYONNEMENT X}

\subsection{Effet de la longueur d'onde}

Les mécanismes d'absorption de l'énergie laser que l'on peut évoquer pour expliquer le chauffage des électrons au sein du nanoplasma dépendent tous fortement de la longueur d'onde incidente. En effet, si l'on considère un chauffage par oscillation des électrons dans le champ électrique, l'énergie pondéromotrice acquise par un électron décroît d'un facteur 4 lorsque l'on change la longueur d'onde du laser de $800 \mathrm{~nm}$ à $400 \mathrm{~nm}$. Dans cette hypothèse, le seuil en éclairement en UV doit donc être plus grand qu'en IR. Par contre, dans le cas d'absorption par bremsstrahlung inverse, plus la longueur d'onde est courte et plus le chauffage des électrons est efficace, ce qui pourrait se traduire, par exemple, par un seuil en éclairement plus faible ou bien par une augmentation du taux de photons X. Nous avons donc étudié précisément le rayonnement $X$, en fonction de la longueur d'onde laser : en IR $(800 \mathrm{~nm})$ et en UV (400 nm obtenu grâce à un cristal doubleur de KDP).

Les résultats obtenus semblent indiquer une faible dépendance du taux absolu d'X en fonction de la longueur d'onde. En particulier, le taux d'X enregistré dans des conditions expérimentales identiques (même système de focalisation) et pour un éclairement similaire, est quasiment le même en UV qu'en IR ainsi que la distribution des espèces ioniques dont est issu le rayonnement $X$. D'autre part, le seuil de 
ainsi que la distribution des espèces ioniques dont est issu le rayonnement $X$. D'autre part, le seuil de production d'X mesuré pour une longueur d'onde de $400 \mathrm{~nm}$ est plus faible (vers $5.10^{14} \mathrm{~W} / \mathrm{cm}^{2}$ ) que pour une longueur d'onde de $800 \mathrm{~nm}$ (entre 1 et $1,5.10^{15} \mathrm{~W} / \mathrm{cm}^{2}$ ). Ce résultat est donc en contradiction avec une image du chauffage des électrons uniquement par oscillation dans le champ laser. En effet, l'énergie d'oscillation des électrons à cet éclairement seuil en UV est de l'ordre de $13 \mathrm{eV}$; ce qui est beaucoup trop faible pour pouvoir ensuite ioniser en couche interne les ions présents au sein du nanoplasma par des collisions de type $\mathrm{e}^{*} /$ ions.

\subsection{Effet de la polarisation}

L'évolution du nanoplasma produit lors de l'interaction du laser de puissance avec le jet d'agrégats est également susceptible d'être modifié en fonction de la polarisation du rayonnement excitateur incident. Les premières études réalisées ont déjà montré, en spectroscopie basse résolution, que l'émission de photons est indépendante de l'orientation relative de la polarisation linéaire de l'onde laser (horizontale ou verticale). Par contre, entre polarisation circulaire et linéaire, on peut avoir, au travers des différents modèles, un certain nombre de variations :

- lors de la première étape d'ionisation par effet de champ, le taux d'ionisation par effet tunnel est 2 fois plus faible avec une polarisation circulaire qu'avec une polarisation linéaire en ce qui concerne les électrons externes ;

- l'énergie pondéromotrice acquise par les électrons soumis à un champ laser circulaire est 2 fois plus importante qu'en polarisation linéaire ;

- enfin, les trajectoires des électrons sont également différentes en présence d'un champ électrique ayant une polarisation linéaire ou circulaire ce qui doit induire des fréquences de collisions différentes ;

Les études que nous avons menées, en spectroscopie basse résolution, n'indiquent pas d'écart significatif dans le taux absolu de photons $\mathrm{X}$ entre une polarisation circulaire et une polarisation linéaire. Par ailleurs, tout comme en fonction de la longueur d'onde, les spectres haute résolution sont quasiment les mêmes lorsque l'on change la polarisation; à ceci près qu'il y a un peu moins (inférieur à $20 \%$ ) d'ions de faibles états de charge $\left(\mathrm{Ar}^{12+}, \mathrm{Ar}^{13+}\right)$. Le rôle de la polarisation du champ incident apparaît donc beaucoup moins importante lors de l'interaction laser - agrégats que, par exemple dans le cas d'un atome unique en champ laser intense.

\section{DISCUSSION ET PERSPECTIVE}

Les résultats expérimentaux que nous avons obtenus ne sont pas, pour l'instant, interprétés par les modèles généralement mis en avant pour expliquer l'interaction d'un laser de puissance avec des agrégats de gaz rare. En particulier, la présence d'un seuil de production de photons $\mathrm{X}$, et donc d'ions d'état de charge élevé possédant des lacunes en couche interne, si bas (vers $10^{15} \mathrm{~W} / \mathrm{cm}^{2}$ ) reste difficilement compréhensible. De même, alors que les mécanismes responsables de l'émission $\mathrm{X}$ (ionisation d'électrons externe - chauffage des électrons - création de lacunes en couches interne) sont fortement dépendant de la longueur d'onde et de la polarisation, des faibles différences ont été observées tant sur le plan quantitatif, via le taux absolu, que sur le plan de la distribution ionique du plasma dont est issu le rayonnement $\mathrm{X}$. Un effort important de modélisation doit donc être réalisé afin de comprendre la compétition entre les différents mécanismes possibles de chauffage des électrons en fonction des paramètres gouvernant l'interaction.

\section{Références}

1. Shao et al., Phys. Rev. Lett. 77, 3343 (1996)

2. Ditmire et al., Nature 386, 55 (1997)

3. McPherson et al., Nature 370, 631 (1994)

4. Rozet et al., Physica Scripta T92, 113 (2001)

5. Lamour et al., Proc. of SPIE, Eds G.A.Kyrala and J.C. Gauthier, 4506, 97 (2002)

6. Hagena et al., J. Chem. Phys. 56, 1793 (1972)

7. Ditmire et al., Phys. Rev. A 53, 3379 (1996) 